\title{
Control of T1DM via Tensor Product-based framework
}

\author{
György Eigner*, Péter Pausits ${ }^{\dagger}$ and Levente Kovács* \\ ${ }^{*}$ Research and Innovation Center of Óbuda University, \\ Physiological Controls Group, Óbuda University, Budapest, Hungary \\ Email: \{eigner.gyorgy,kovacs.levente\}@nik.uni-obuda.hu \\ ${ }^{\dagger}$ Research and Innovation Center of Óbuda University, \\ Antal Bejczy Center for Intelligent Robotics, Óbuda University, Budapest, Hungary \\ Email: peter.pausits@irob.uni-obuda.hu
}

\begin{abstract}
With this study our goal was to investigate and prove the usability of the Tensor Product (TP)-transformation based modeling and control regarding the control of Diabetes Mellitus (DM). In details, we examined the TP-based modeling possibilities of Type $1 \mathrm{DM}$ (T1DM) by using the simple well-known Minimal Model. We provided a TP-based controller design solution, where the main focus was on the disturbance rejection. Generally, the TP-based framework using the Parallel Distributed Control (PDC)- and Linear Parameter Varying (LPV) theorems via wellconditioned Linear Matrix Inequalities (LMI) to realize the TPbased controller. In this manner the basis of our controller design procedure was the development of the control oriented qLPV model form, the selection and application of appropriate LMIs through which the PDC-based TP controller can be developed and the realization of the control environment. We prove the usability of the developed control solution on the mentioned T1DM model beside unfavorable disturbances.
\end{abstract}

\section{INTRODUCTION}

The benefits of physiological modeling and control are unquestionable these days [1]. Modern advanced control solutions can be found in several medical applications, for example regulation of anesthesia [2] or control of Blood Glucose (BG) level [3].

Computer aided modeling and control is definitely important in case of DM, where the goal is to keep the BG level in a tight range. However, researchers on the field have to face with several challenges: the human metabolic system is highly nonlinear, varies patient-by-patient and contains several time-delay processes. These circumstances require advanced modeling and control methodologies, if the aim is to reach high quality control with appropriate performances [1], [3], [4].

In the recent years, highly developed approaches appeared regarding to modeling and control of DM [3]. Beside the evolution of the commonly used Model Predictive Control (MPC)-based advanced solutions [5], [6], the Linear Parameter Varying (LPV) techniques [7], Linear Matrix Inequality

Gy. Eigner was supported by the ÚNKP-16-3/IV. New National Excellence Program of the Ministry of Human Capacities. This project has received funding from the European Research Council (ERC) under the European Union's Horizon 2020 research and innovation programme (grant agreement No 679681).
(LMI)-based methodologies [8] and the combination of these [9] became determining in this research field [3]. One of these new solutions is the TP model transformation based modeling and controller design which successfully combines the mentioned LPV and LMI techniques [10].

In this paper, we investigated the modeling and control possibility of the well-known T1DM model (Minimal Model) via TP framework.

The paper is structured as follows: first, we introduce the TP model transformation. After, we present the used T1DM model, the applied qLPV and TP forms of it. Further, we demonstrate a possible way for the controller design via LMIs. Finally, we present the results and the conclusions to sum up the observations regarding the developed controller solution.

\section{Tensor Product Model Transformation}

The TP model transformation has strong connection to the Fuzzy methodologies - in sort of a way, the TP theorem can be handled as a special extended form of the Fuzzy theorem [10].

TP model transformation of a given function results a TP model function which approximates the original function [11], [12]. The qLPV models can be handled as qLPV functions, thus the TP model transformation of them is possible. The resulting TP model form is able to approximate the original qLPV model with given accuracy determined by the predefined properties (mainly the sampling density of the parameter space and the retained singular values of the core tensor before simplification) of the TP model transformation. Moreover, the TP model transformation can be combined with LMIbased controller design techniques through the convex hull manipulations of polytopic structures [10].

Definition 1 - State Space (SS) form of a qLPV model: a given qLPV model can be described with its SS representation via system matrix $\mathbf{S}(\mathbf{p}(t))$ in compact form:

$$
\begin{gathered}
\dot{\mathbf{x}}(t)=\mathbf{A}(\mathbf{p}(t)) \mathbf{x}(t)+\mathbf{B}(\mathbf{p}(t)) \mathbf{u}(t)+\mathbf{E}(\mathbf{p}(t)) \mathbf{r}(t) \\
\mathbf{y}(t)=\mathbf{C}(\mathbf{p}(t)) \mathbf{x}(t)+\mathbf{D}(\mathbf{p}(t)) \mathbf{u}(t)+\mathbf{D}_{\mathbf{2}}(\mathbf{p}(t)) \mathbf{v}(t) \\
\mathbf{S}(\mathbf{p}(t))=\left(\begin{array}{lll}
\mathbf{A}(\mathbf{p}(t)) & \mathbf{B}(\mathbf{p}(t)) & \mathbf{E}(\mathbf{p}(t)) \\
\mathbf{C}(\mathbf{p}(t)) & \mathbf{D}(\mathbf{p}(t)) & \mathbf{D}_{2}(\mathbf{p}(t))
\end{array}\right)
\end{gathered}
$$


where $\mathbf{A}(\mathbf{p}(t)) \in \mathbb{R}^{k \times k}$ is the state-, $\mathbf{B}(\mathbf{p}(t)) \in \mathbb{R}^{k \times m}$ is the control input-, $\mathbf{E}(\mathbf{p}(t)) \in \mathbb{R}^{k \times h}$ is the disturbance-, $\mathbf{C}(\mathbf{p}(t)) \in$ $\mathbb{R}^{l \times k}$ is the output-, $\mathbf{D}(\mathbf{p}(t)) \in \mathbb{R}^{l \times m}$ is the forward- and $\mathbf{D}_{\mathbf{2}}(\mathbf{p}(t)) \in \mathbb{R}^{l \times h}$ is the noise-matrix, respectively. The $\mathbf{u}(t) \in$ $\mathbb{R}^{m}, \mathbf{r}(t) \in \mathbb{R}^{h}, \mathbf{y}(t) \in \mathbb{R}^{l}$ and $\mathbf{x}(t) \in \mathbb{R}^{k}$ vectors are the control, disturbance, output and state-vectors, respectively. The system matrix $\mathbf{S}(\mathbf{p}(t)) \in \mathbb{R}^{(k+l) \times(k+m+h)}$ is parameter vector dependent, which equivocally determines the qLPV system. Further, $\mathbf{p}(t) \in \Omega \in \Pi$ is the time dependent parameter vector, where $\Pi \in \mathbb{R}^{N}$ is the $N$ dimensional real parameter space.

Definition 2 - Transformational space $\Omega$ : bounded $N$ dimensional hypercube in the $\Pi$ parameter space, which does determine the minimum and maximum values of the scheduling parameters $\left(p_{i}(t), i:=N\right)$. The parameter vector consist of the scheduling variables which are the terms of the original model. Thus $\mathbf{p}(t): \Omega=\left[p_{1, \min }, p_{1, \max }\right] \times\left[p_{2, \min }, p_{2, \max }\right] \times \ldots \times$ $\left[p_{N, \min }, p_{N, \max }\right] \in \mathbb{R}^{N}$.

Definition 3 - Convex polytopic model with finite elements: describes the $\mathbf{S}(t)$ actual model. The $\mathbf{S}(t)$ is calculated as the convex combination of $\mathbf{S}_{r} \in \mathbb{R}^{(k+l) \times(k+m+h)}$ LTI vertex system inside the $\Omega$ :

$$
\mathbf{S}(\mathbf{p}(t))=\sum_{r=1}^{R} w_{r}(p(t)) \mathbf{S}_{r},
$$

where the convexity requires that the weighting functions $w_{r}(p(t)) \in[0,1]$ and $R$ has to be bounded.

Definition 4 - TP type convex polytopic model with finite elements: describes the actual model $\mathbf{S}(t)$ as the convex combination of the $\mathbf{S}_{r} \in \mathbb{R}^{(k+l) \times(k+m+h)}$ LTI vertex system inside the $\Omega(p(t) \in \Omega)$ :

$$
\mathbf{S}(\mathbf{p}(t))=\sum_{i_{1}=1}^{I_{1}} \sum_{i_{2}=1}^{I_{2}} \ldots \sum_{i_{N}=1}^{I_{N}} \prod_{n=1}^{N} w_{n, i_{n}}\left(p_{n}(t)\right) \mathbf{S}_{i_{1}, i_{2}, \ldots, i_{N}} .
$$

According to [10] the compact notation of (3):

$$
\mathbf{S}(\mathbf{p}(t))=\mathcal{S} \underset{\mathrm{n}=1}{\mathrm{~N}} \mathbf{w}_{n}\left(p_{n}(t)\right)
$$

Here the coefficient (core) tensor $\mathcal{S} \in$ $\mathbb{R}^{I_{1} \times I_{2} \times \ldots \times I_{N} \times(k+l) \times(k+m+h)}$ can be derived from $\mathbf{S}_{i_{1}, i_{2}, \ldots, i_{N}}$ LTI vertex system and the weighting vector $\mathbf{w}_{n}\left(p_{n}(t)\right)$ which consists of the univariate continuous weighting functions $w_{n, i_{n}}\left(p_{n}(t)\right)\left(i_{n}=1 \ldots I_{N}\right)$.

Definition 5 - TP model transformation: a numerical process which transforms given qLPV models in the form of (1a) into TP model form of (4). Several LMI based controller design methodology can be applied accompanied with the provided TP model. The TP model transformation allows the application of convex hull manipulation during the TP-model transformation. Accuracy of the resulting TP model depends on the sampling density of the $\Omega$ (ie. the number of used LTI vertex), specificities of the used HOSVD procedure and the applied TP function. More details can be found in [10].

Definition 6 - The canonical form of the qLPV model (based on given HOSVD method): without using complexity reduction and convex hull manipulation, the resulting TP model becomes equivalent to the numerical reconstruction of the HOSVD of the original qLPV model. Because of the HOSVD is used on the qLPV model, the resulting HOSVD canonical form consists of singular function in an orthonormal structure, further a coefficient or core tensor, which contains system vertices assigned to the higher order singular values. More details can be found in [10].

Definition 7 - Convex TP model: the resulting TP model is convex, if and only if the weighting functions satisfy the following criteria:

$$
\begin{aligned}
& \forall n, i, p_{n}(t): w_{n, i_{n}}\left(p_{n}(t)\right) \in[0,1] \\
& \forall n, p_{n}(t): \sum_{i=1}^{I_{n}} w_{n, i_{n}}\left(p_{n}(t)\right)=1
\end{aligned}
$$

In this study we applied the tight MVS type convex hull, which has several beneficial properties regarding the approximation, used computational capacity and simplification [13], [14].

Definition 8 - MVS-type convex TP model: the following TP model is a MVS type convex model

$$
\mathbf{S}(\mathbf{p})=\mathcal{S} \underset{\mathrm{n}=1}{\mathrm{~N}} \mathbf{w}^{(n)}\left(p_{n}\right),
$$

if the $(S)_{j_{n}=j} n$-mode sub-tensors evolve a minimal volume bounding simplex for $\mathcal{S} \times_{n} \mathbf{w}_{j n}^{(n)}\left(p_{n}\right)$ trajectory over $n=1 . . N$ for the $S \in \mathbb{S}^{J_{1} \times \ldots \times J_{N}}$ core tensor, which is realized from the $\mathbf{S}_{j_{1}, \ldots, j_{N}}$ matrices.Detailed explanations, derivations, examples and case studies can be found in [10], [13]-[17]. We utilized the TP Toolbox ${ }^{\circledR}$ in this study. The toolbox is a MATLAB based tool and means a convenient and effective possibility to realize the TP based approached.

\section{USED T1DM MODEL}

In this study we have used a special form of the well-known Minimal Model derived in [18]:

$$
\begin{gathered}
\dot{G}(t)=-\left(p_{1}+X(t)\right) G(t)+p_{1} G_{B}+\frac{d(t)}{V_{G}} \\
\left.\dot{X}(t)=-p_{2} X(t)\right)+p_{3}\left(I(t)-I_{B}\right) \\
\dot{I}(t)=-n\left(I(t)-I_{B}\right)+\frac{u(t)}{V_{I}}
\end{gathered}
$$

The model consist of three states: blood glucose concentration $G(t)$ in [mg/dL], which is the output of the model as well; the insulin-excitable tissue glucose uptake activity $X(t)$ in $[1 / \mathrm{min}]$; and the blood insulin concentration $I(t)$ in $[\mu \mathrm{U} / \mathrm{mL}]$. The inputs of the model are the insulin $(u(t)[\mu \mathrm{U} / \mathrm{mL} / \mathrm{min}])$ and the (glucose intake $d(t)[\mathrm{mg} / \mathrm{dL} / \mathrm{min}]$ ), respectively.

We have used the following dataset in this research: $G_{b}=$ $110 \mathrm{mg} / \mathrm{dL}, I_{b}=1.5 \mu \mathrm{U} / \mathrm{mL}, p_{1}=0.0281 / \mathrm{min}, p_{2}=0.025$ $1 / \mathrm{min}, p_{3}=0.00013 \mathrm{~min}^{-2} /(\mu \mathrm{U} / \mathrm{mL}), n=0.231 / \mathrm{min}, h=130$ $\mathrm{mg} / \mathrm{dL}, \gamma=0.01(\mu \mathrm{U} / \mathrm{mL}) /(\mathrm{mg} / \mathrm{dL}) / \mathrm{min}, V_{G}=120 \mathrm{dL}$ and $V_{I}=$ $90 \mathrm{dL}$. The parameter set belongs to a real subject detailed in [18]. 


\section{CONTROL ORIENTED QLPV- AND TP-MODEL FORM}

In this study we used PDC theorems via LPV and LMI frameworks regarding the TP-based control. In case of state feedback-based PDC controller a plausible solution is the usage of control oriented model form which means deviation based modeling [10]. In this case the control oriented qLPV model describe not just the dynamics of the process (via states), but also the deviation from a possible model equilibrium (error dynamics). Thus, the control goal is to avoid or eliminate the deviation from this model equilibrium: $\Delta \mathbf{x}(t)=\mathbf{x}(t)-\mathbf{x}_{d}$, where $\mathbf{x}_{d}$ belongs to a given permanent state vector.

A possible model equilibrium can be calculated by using the steady-state conditions. We selected $G_{d}=90$ and $u_{d}=0$ as steady-state values $(90 \mathrm{mg} / \mathrm{dL}$ is a healthy blood glucose concentration and $u_{d}=0$ does mean that there is no external insulin intake). Further, we considered that $G_{d} \neq G_{B}$ - that means we did not apply the model equilibrium $G_{B}$, but an "enforced" equilibrium. The steady-state values can be calculated by rearranging $(7 \mathrm{a})-(7 \mathrm{c})$ :

$$
\begin{gathered}
I_{d}=I_{b}+\frac{u_{d}}{V_{I} n}=I_{b} . \\
X_{d}=\frac{p_{3}}{p_{2}} I_{d} . \\
d_{d}=\left(p_{1}+X_{d}\right) G_{d}-p_{1} G_{B} .
\end{gathered}
$$

The control oriented (deviation-based) model can be derived by using the model equations and the calculated steady-state values. It should be noticed that more than one control oriented model form can be derived. However, in real life only $G(t)$ can be measured - in the light of this fact we used that form, where only $G(t)$ appears in the $\mathbf{p}(t)$.

First, $\Delta G(t)$ can be calculated in the following way:

$$
\begin{aligned}
& \Delta \dot{G}(t)=\dot{G}(t)-0= \\
& -\left(p_{1}+X(t)\right) G(t)+p_{1} G_{B}+d(t)- \\
& {\left[-\left(p_{1}+X_{d}\right) G_{d}+p_{1} G_{B}+d_{d}\right]=} \\
& -p_{1}\left(G(t)-G_{d}\right)+\left(d(t)-d_{d}\right)-X(t) G(t)+X_{d} G_{d}= \\
& -p_{1} \Delta G(t)+\Delta d(t)- \\
& X(t) G(t)+X_{d} G_{d}+X_{d} G(t)-X_{d} G(t) \\
& \Delta \dot{G}(t)=-\left(p_{1}+X_{d}\right) \Delta G(t)-G(t) \Delta X(t)+\frac{1}{V_{G}} \Delta d(t)
\end{aligned}
$$

The remaining $\Delta$ states can be calculated similarly (due to the lack of space we did not detail here).

$$
\Delta \dot{X}(t)=-p_{2} \Delta X(t)+p_{3} \Delta I(t)
$$

and

$$
\Delta \dot{I}(t)=-n \Delta I(t)+\frac{1}{V_{I}} \Delta u(t) .
$$

In order to use the TP model transformation as detailed above the compact SS form of qLPV model should be realized. Since, the deviation-based states are $\Delta \mathbf{x}(t)=$ $[\Delta G(t), \Delta X(t), \Delta I(t)]^{T}$ and the compact SS form becomes:

$$
\begin{aligned}
& {\left[\begin{array}{l}
\Delta \dot{\mathbf{x}}(t) \\
\Delta y(t)
\end{array}\right]=\left[\begin{array}{ccccc}
-\left(p_{1}+X_{d}\right) & -G(t) & 0 & 0 & \frac{1}{V_{G}} \\
0 & -p_{2} & p_{3} & 0 & 0 \\
0 & 0 & -n & \frac{1}{V_{I}} & 0 \\
1 & 0 & 0 & 0 & 0
\end{array}\right]\left[\begin{array}{c}
\Delta \mathbf{x}(t) \\
\Delta u(t) \\
\Delta r(t)
\end{array}\right] .} \\
& {\left[\begin{array}{l}
\Delta \mathbf{x}(t) \\
\Delta y(t)
\end{array}\right]=\mathbf{S}(\mathbf{p}(t))\left[\begin{array}{l}
\Delta \mathbf{x}(t) \\
\Delta u(t) \\
\Delta r(t)
\end{array}\right]}
\end{aligned}
$$

By applying the TP model transformation on (14) the general TP model structure becomes $\mathbf{S}(G(t))=\mathcal{S} \times \mathbf{w}(G(t))$.

The variation of the obtained MVS type weighting functions can be seen on Fig. 1. (the resulting weighting functions are linear).

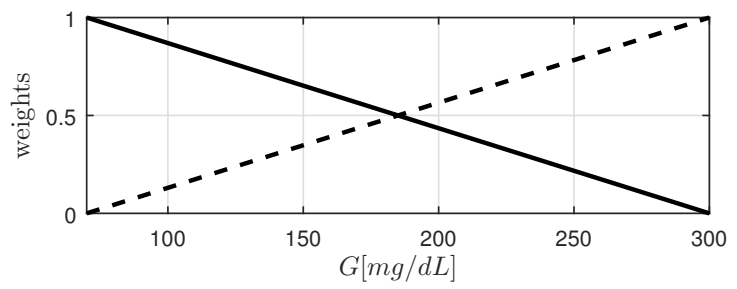

Figure 1. Weighting functions of the TP polytopic model

The resulting TP model form in this case became:

$$
\mathbf{S}(G(t))=\mathcal{S} \underset{\mathrm{n}=1}{\mathbb{1}} \mathbf{w}_{n}\left(p_{n}(t)\right)=\mathcal{S} \times_{1} \mathbf{w}_{1}(G(t))
$$

\section{Controller Design via LMis}

Our goal was to prove the usability of TP-based controller design regarding the control of T1DM. Therefore, we applied common state feedback-based PDC theorems via LMI-based methods. In this manner the goal of the controller design to find those feedback gains $\mathbf{F}_{i}$ which satisfy the predefined requirements and appropriate for the LTI systems $\mathbf{S}_{i}$ in the vertices. The control signal in state feedback control gets the following form in this given case:

$$
u(t)=-\mathcal{F} \underset{\mathrm{n}=1}{\mathbb{\bigotimes}} \mathbf{w}_{n}\left(p_{n}(t)\right) \mathbf{x}(t)=-\mathcal{F} \times_{1} \mathbf{w}_{1}(G(t)) \mathbf{x}(t)
$$

There are several LMI theorems available for controller design to derive the feedback gain tensor $\mathcal{F}$ of the controller. Since, the glucose intake has to be handled as disturbance from the system point of view, we applied disturbance rejection during the controller design. Moreover, we used constraint on the control input - in order to avoid physiologically irrelevant or not tolerable control signals. 
Theorem 1 - Consider that the initial condition $\mathbf{x}(0)$ is known. The constraint on control input $\|\mathbf{u}(t)\|_{2} \leq \mu$ is enforced at all times $t \geq 0$ if the LMIs

$$
\left[\begin{array}{cc}
1 & \mathbf{x}(0)^{T} \\
\mathbf{x}(0)^{T} & \mathbf{X}
\end{array}\right] \geq \mathbf{0}
$$

and

$$
\left[\begin{array}{cc}
\mathbf{X} & \mathbf{M}_{i}^{T} \\
\mathbf{M}_{i} & \mu^{2} \mathbf{I}
\end{array}\right] \geq \mathbf{0}
$$

where $\mathbf{X}=\mathbf{P}^{-1}$ and $\mathbf{M}_{i}=\mathbf{F}_{i} \mathbf{X}$. Proof can be found in [19].

Because of the initial conditions are not known in most of cases because of the specificities of the problem, we replaced the (17) with an initial state independent conditions, which is able to hold the validity of (18).

Theorem 2 - Consider that the control input $\|\mathbf{u}(t)\|_{2} \leq \phi$, where $\mathbf{x}(0)$ is unknown, although the upper bound $\phi$ is known. In this case:

$$
\mathbf{x}^{T}(0) \mathbf{X}^{-1} \mathbf{x}(0) \leq 1 \text { if } \phi^{2} \mathbf{I} \leq \mathbf{X},
$$

where $\mathbf{X}=\mathbf{P}^{-1}$ [19]. Therefore (17) can be replaced to (19).

Disturbance rejection can be realize in case of a continuous TP model by minimizing the effect of $\mathbf{r}(t)$ disturbance on the measured (or performance) output $\mathbf{y}(t)$ as

$$
\sup _{\|\mathbf{r}(t)\|_{2} \neq 0} \frac{\|\mathbf{y}(t)\|_{2}}{\|\mathbf{r}(t)\|_{2}} \leq \gamma .
$$

Theorem 3 - In case of continuous systems, the feedback gains $\mathbf{F}_{i}$ which stabilize the TP model and minimize $\gamma$ in (20) occurs via solving the following LMI minimization problem:

$$
\begin{aligned}
& \underset{\mathbf{x}, \mathbf{M}_{1}, \ldots, \mathbf{M}_{r}}{\operatorname{minimize}} \gamma^{2} \\
& \text { subject to } \\
& \mathbf{X}>\mathbf{0}
\end{aligned}
$$

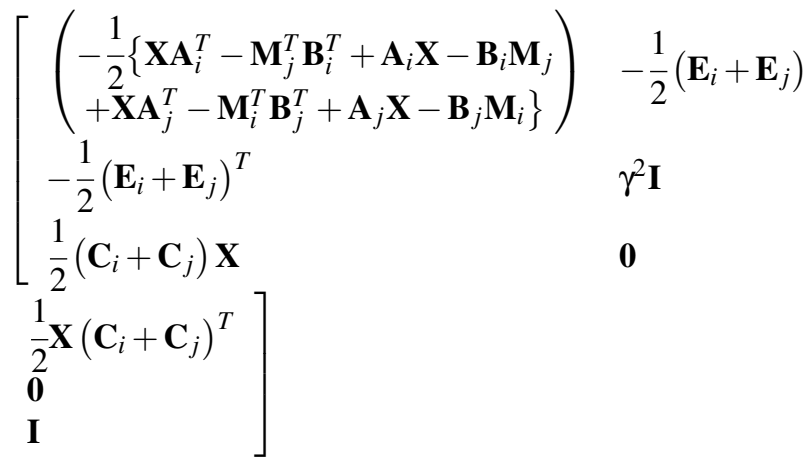

where $\mathbf{M}_{i}=\mathbf{F}_{i} \mathbf{X}$. Proof can be found in [19].

We have used MOSEK Apps. solver [20] and the YALMIP toolbox [21] in order to calculate the appropriate feedback gains via the LMIs above.

During the research we consider the following parameters: the bounded hypercube was equal to $\Omega=[70,300]$ and the sampling density in the parameter domain (number of grid points) was equal to $n=301$; moreover, the parameters of the LMIs above were: $\phi=20$ and $\mu=0.031$.

Finally, the following feedback gains occurred:

$$
\mathbf{F}_{1}=\left[\begin{array}{lll}
0.7042 & -926.3393 & -0.3088
\end{array}\right]
$$

and

$$
\begin{gathered}
\mathbf{F}_{2}=\left[\begin{array}{lll}
0.0010 & -1.2199 & -0.0004
\end{array}\right] 10^{3} \\
\text { VI. RESULTS }
\end{gathered}
$$

We have tested the developed controller solution beside two different feed-intake protocols:

1) Protocol 1.: Unfavorable, high, symmetric pulse-kind CHO intake. $100 \mathrm{~g}$ carbohydrate $(\mathrm{CHO})$ in every pulses with the following settings: pulse width: $10 \%$ of the period of time, amplitude: 5, period of time: 200 .

2) Protocol 2.: Real, randomized CHO intake based on the daily requirement of an adult according to the WHO definitions [22]. In details, the main meals (breakfast, lunch, dinner) contains the $75 \%$ of the daily $\mathrm{CHO}$ consumption, the other meals contains the rest $25 \%$. The sum of the amount of $\mathrm{CHO}$ in meals is equal to the total needs (we calculated with $1700 \mathrm{kcal}$ which means $425 \mathrm{~g}$ daily $\mathrm{CHO}$ intake). We applied 5 different meals (two smaller $\mathrm{CHO}$ intake were considered between the breakfast and lunch and between the lunch and the dinner).

In order to make the feed intake realistic we completed the Minimal Model with an absorption subsystem from [23]. The subsystem was not embedded into the model structure, however, in order to avoid the unrealistic behavior of the Miminal Model (which can occur in case of impulse kind glucose input) the usage of it was necessary. The absorption subsystem can be described with the following equations:

$$
\begin{aligned}
& \dot{D}_{1}(t)=1000 A_{G} p(t)-\frac{D_{1}(t)}{\tau_{D}} \\
& \dot{D}_{2}(t)=\frac{D_{1}(t)}{\tau_{D}}-\frac{D_{2}(t)}{\tau_{D}} \\
& d(t)=\frac{D_{2}(t)}{\tau_{D}}
\end{aligned},
$$

where $p(t) \mathrm{g}$ is the glucose intake, $\tau_{D}=40 \mathrm{~min}$ the absorption time, $A_{G}=0.8$ is the glucose utilization.

The simulation time was $900 \mathrm{~min}$ in each case.

\section{A. Results in case of Protocol 1.}

Figure 2. shows the simulation results in case of Protocol 1.

The first diagram represents the state vector of the original nonlinear model during operation. It can see that after the initial transients the states oscillates symmetrically accordingly the applied feed intake. Hypoglycemia was totally avoided (the BG level varied between 178 and $87 \mathrm{mg} / \mathrm{dL}$ ). 
The second diagram shows the state vector belongs to the TP model - the properties of the TP states are the same as in the previous (original nonlinear model) case.

The deviation between the original and TP model can be seen on the third diagram - which represents the 2-norm based error of the states belong to original and TP models. Only numerical error occurred (the magnitude was $10^{-13}$ ).

The fourth diagram presents the injected external insulin determined by the developed controller. Naturally, because the basis was the state feedback, the same oscillation can be seen as in the previous cases. Namely, after the initial transients, the controller injects as many insulin which can compensate the glucose intake and keeps the BG level in normal ratio.

The last diagram represents the rate of appearance of the absorbed glucose provided by the absorption subsystem (according to (24)). The vary of $d(t)$ determines the behavior of every signals, since the goal of the control is the rejection of the effect of the disturbance $(d(t))$ on the controlled output $(G(t))$ through the control signal $(u(t))$.

It can be concluded that the controller is able to handle the BG level effectively, totally avoid the hypoglycemia and allows a moderated natural hyperglycemia after the feed intakes, which disappears quickly.
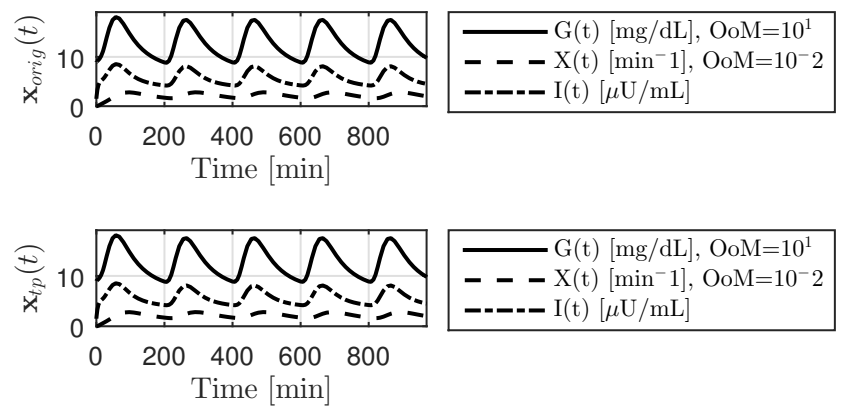

$\longrightarrow \mathrm{G}(\mathrm{t})[\mathrm{mg} / \mathrm{dL}], \mathrm{OoM}=10^{1}$ - - - X $(\mathrm{t})\left[\mathrm{min}^{-} 1\right], \mathrm{OoM}=10^{-} 2$ ---- I $\mathrm{I}(\mathrm{t})[\mu \mathrm{U} / \mathrm{mL}]$
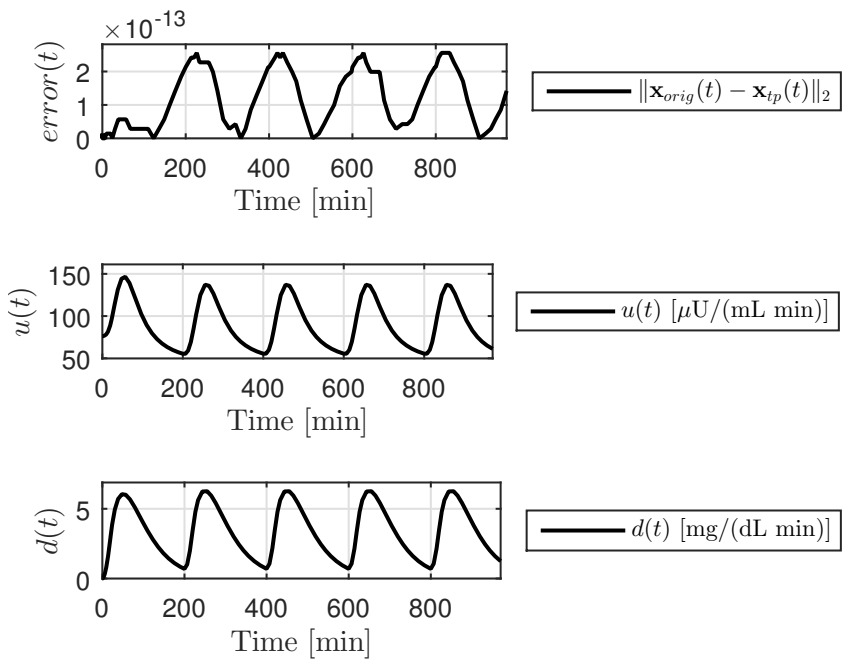

Figure 2. Simulation results in case of Protocol 1. [OoM - Order of Magnitude]

\section{B. Results in case of Protocol 2.}

Figure 3. shows the simulation results in case of Protocol 2.

The sequence and meaning of the diagrams on Fig. 3. is the same as in Sec. VI-A.

The BG level varied between 186 and $81 \mathrm{mg} / \mathrm{dL}$ under the total simulation time (as it can see on the first diagram).

The TP model perfectly mimic the original nonlinear model (second diagram) - which strengthened by the third diagram which proves only small deviation occurred between the states of the original and TP models (the order of magniute was $\left.10^{-13}\right)$.

The fourth diagram represents the injected insulin which act upon the needs of the system (avoid the effect of disturbance input on the controlled output). Thus, the shape of the control signal in time is similar to the last diagram (the disturbance).

The last diagram presents the feed intake (the disturbance) over time. It is visible that the rate of appearance of the absorbed glucose (which is provided by the absorption subsystem according to (24)) is act upon the needed CHO intake of an adult divided into five bigger meals based on Protocol 2.

These glucose load is much natural than the introduced one in Protocol 1. It can see that the developed controller effectively handles the daily glucose load and the hypoglycemia is totally avoided. The maximum BG level during the simulation was $186 \mathrm{mg} / \mathrm{dL}(10.33 \mathrm{mmol} / \mathrm{L})$ which means a natural and short hyperglycemia after the "biggest" meal (after lunch).

\section{CONCLUSiON AND FURTHER WORK}

It can be concluded that the developed TP-based controller solution effectively handles the BG level in the given circumstances. We have tested the realized controller based on two feed intake protocols.

The first one was unfavorable, high, however, symmetric pulse-kind glucose load. The controller can deal with the disturbance well. The hypoglycemia was totally avoided and only moderated hyperglycemia occurred which disappeared quickly.

The second protocol (based on the WHO's prescription for an adult) described a usual daily glucose load. In this case, the controller avoids the hypoglycemia and only one higher hyperglycemic period occurred after the "biggest" meal (after lunch), however, the controller reacts to it immediately and the high BG level quickly decays.

In our future work we will test the developed controller designing method in case of complex T1DM models. Moreover, we are going to embed other LMIs: $\mathcal{H}_{\infty}$-based method completed with observer design.

\section{ACKNOWLEDGMENT}

Gy. Eigner and P. Pausits thankfully acknowledge the support of the Robotics Special College and the Doctoral School of Applied Informatics and Applied Mathematics of Óbuda University. 

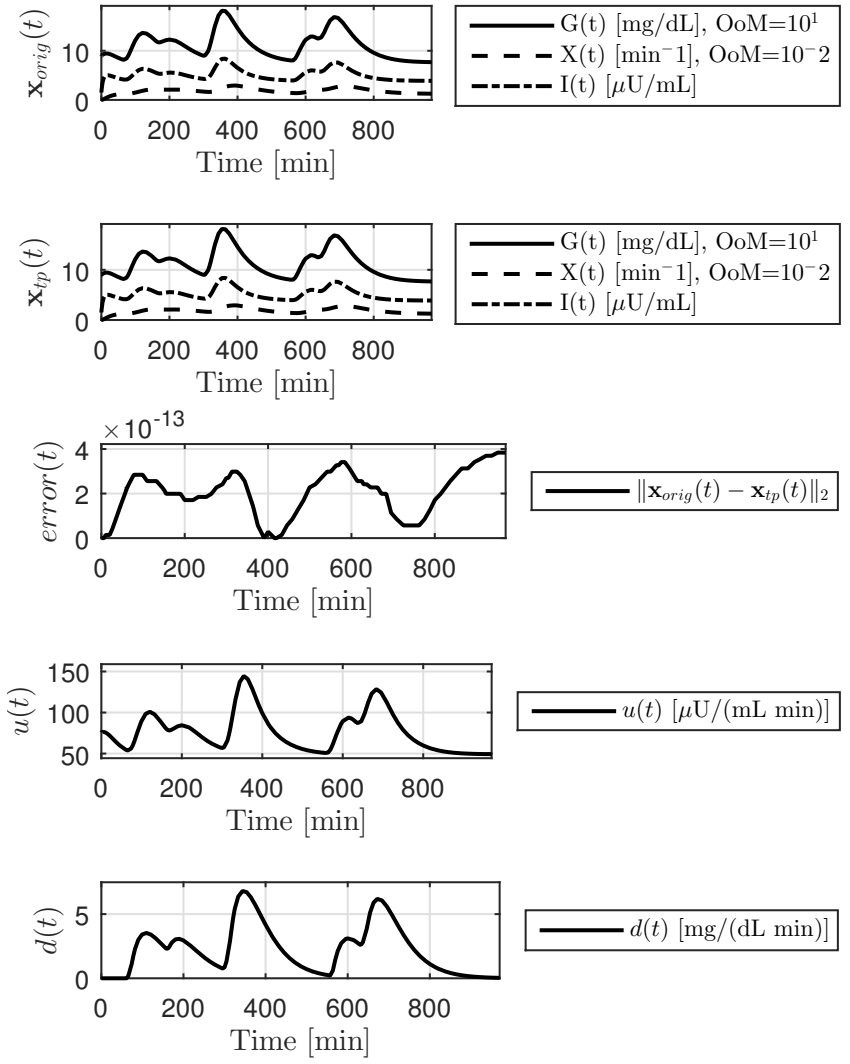

Figure 3. Simulation results in case of Protocol 2. [OoM - Order of Magnitude]

\section{REFERENCES}

[1] J. Bronzino and D. Peterson, The Biomedical Engineering Handbook, 4th ed. Boca Raton, Florida, USA: CRC Press, 2015.

[2] C. Ionescu, D. Copot, and R. De Keyser, "Modelling for control of depth of hypnosis: a patient friendly approach," in Proceedings of the 2016 IEEE International Conference on Systems, Man, and Cybernetics. IEEE SMC, 2016, pp. 2653 - 2658.

[3] A. Haidar, "The artificial pancreas: How closed-loop control is revolutionizing diabetes," IEEE Contr Syst Mag, vol. 36, pp. 28 - 47, 2016.

[4] M. Messori, C. Cobelli, and L. Magni, "Artificial pancreas: from insilico to in-vivo," in 9th International Symposium on Advanced Control of Chemical Processes. IFAC, 2014, pp. 1301 - 1309.

[5] M. Naerum, "Model predictive control for insulin administration in people with type 1 diabetes," Technical University of Denmark, Tech. Rep., 2010.

[6] V.N. Shah, A. Shoskes, B. Tawfik, and S.K. Garg, "Closed-loop system in the management of diabetes: Past, present, and future," Diabetes Technol The, vol. 16, no. 8, pp. 477-490, 2014.

[7] Gy. Eigner, J.K. Tar, I. Rudas, and L. Kovacs, "LPV-based quality interpretations on modeling and control of diabetes," ACTA Pol Hung, vol. 13, no. 1, pp. $171-190,2016$.

[8] P. Palumbo, S. Ditlevsen, A. Bertuzzi, and A. de Gaetano, "Mathematical modeling of the glucose-insulin system a review," Math Biosci, vol. 244, no. 2, pp. 69-81, 2013.

[9] P. Szalay, Gy. Eigner, and L. Kovacs, "Linear matrix inequality-based robust controller design for type-1 diabetes model," in IFAC 2014 - 19th World Congress of The International Federation of Automatic Control. IFAC, 2014, pp. $9247-9252$.

[10] P. Baranyi, Y. Yam, and P. Varlaki, Tensor Product Model Transformation in Polytopic Model-Based Control, 1st ed., ser. Series: Automation and Control Engineering. Boca Raton, USA: CRC Press, 2013.
[11] P. Baranyi, D. Tikk, Y. Yam, and R. Patton, "TP model transformation as a way to lmi-based controller design," Comput Ind, vol. 51, no. 3, pp. $281-297,2003$.

[12] P. Baranyi, "TP model transformation as a way to lmi-based controller design," IEEE T Ind Electron, vol. 51, no. 2, pp. 387 - 400, 2004.

[13] J. Kuti, P. Galambos, and P. Baranyi, "Minimal volume simplex (mvs) approach for convex hull generation in tp model transformation," in 2014 18th International Conference on Intelligent Engineering Systems (INES 2014). IEEE Hungary Section, 2014, pp. 187 - 192.

[14] - "Minimal volume simplex (MVS) polytopic model generation and manipulation methodology for tp model transformation," Asian $J$ Control, vol. 19, no. 1, pp. 1 - 13, 2017.

[15] P. Galambos and P. Baranyi, " $\mathrm{TP}_{\tau}$ model transformation: A systematic modelling framework to handle internal time delays in control systems," Asian J Control, vol. 17, no. 2, pp. 1 - 11, 2015.

[16] B. Takarics and P. Baranyi, "TP model-based robust stabilization of the 3 degrees-of-freedom aeroelastic wing section," ACTA Polytech Hung, vol. 12 , no. 1 , pp. $209-228,2015$.

[17] _ - "Friction compensation in tp model form - aeroelastic wing as an example system," ACTA Polytech Hung, vol. 12, no. 4, pp. $127-145$, 2015.

[18] F. Chee and T. Fernando, Closed-Loop Control of Blood Glucose. Heidelberg, Germany: Springer, 2007.

[19] K. Tanaka and H. O. Wang, Fuzzy Control Systems Design and Analysis: A Linear Matrix Inequality Approach, 1st ed. Chichester, UK: John Wiley and Sons, 2001

[20] MOSEK ApS, The MOSEK optimization toolbox for MATLAB manual. Version 7.1 (Revision 28)., 2015. [Online]. Available: http://docs.mosek.com/7.1/toolbox/index.html

[21] J. Löfberg, "YALMIP : A Toolbox for Modeling and Optimization in MATLAB," in In Proceedings of the CACSD Conference, Taipei, Taiwan, 2004

[22] World Health Organization, Food and Agriculture Organization of the United Nations, United Nations University, Human energy requirements, 1st ed. Rome, Italy: WHO, 2004.

[23] R. Hovorka, F. Shojaee-Moradie, P.V. Carroll, L.J. Chassin, I.J. Gowrie, N.C. Jackson, R.S. Tudor, M. Umpleby, and D.H. Jones, "Partitioning glucose distribution/transport, disposal, and endogenous production during ivgtt," Am J Physiol Endocrinol Metab, vol. 282, no. 5, pp. E992 1007, 2002. 